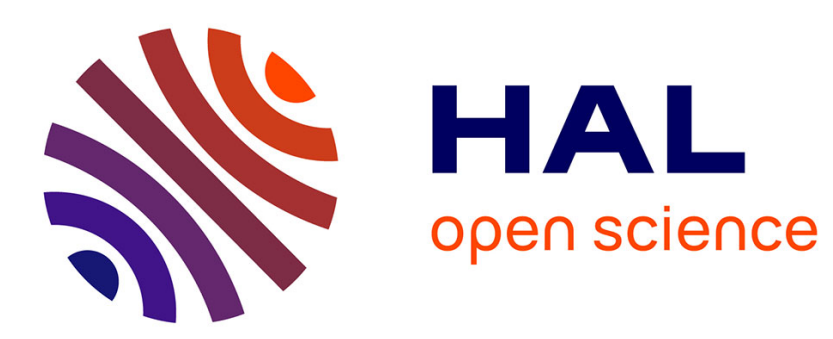

\title{
Unsupervised Bayesian decomposition of multiunit EMG recordings using Tabu search.
}

\author{
Di Ge, Eric Le Carpentier, Dario Farina
}

\section{To cite this version:}

Di Ge, Eric Le Carpentier, Dario Farina. Unsupervised Bayesian decomposition of multiunit EMG recordings using Tabu search.. IEEE Transactions on Biomedical Engineering, 2010, 57 (3), pp.561-71. 10.1109/TBME.2009.2022277 . inserm-00954651

\section{HAL Id: inserm-00954651 https://www.hal.inserm.fr/inserm-00954651}

Submitted on 3 Mar 2014

HAL is a multi-disciplinary open access archive for the deposit and dissemination of scientific research documents, whether they are published or not. The documents may come from teaching and research institutions in France or abroad, or from public or private research centers.
L'archive ouverte pluridisciplinaire HAL, est destinée au dépôt et à la diffusion de documents scientifiques de niveau recherche, publiés ou non, émanant des établissements d'enseignement et de recherche français ou étrangers, des laboratoires publics ou privés. 


\title{
Unsupervised Bayesian Decomposition of Multiunit EMG Recordings Using Tabu Search
}

\author{
Di Ge, Eric Le Carpentier, and Dario Farina*, Senior Member, IEEE
}

\begin{abstract}
Intramuscular electromyography (EMG) signals are usually decomposed with semiautomatic procedures that involve the interaction with an expert operator. In this paper, a Bayesian statistical model and a maximum a posteriori (MAP) estimator are used to solve the problem of multiunit EMG decomposition in a fully automatic way. The MAP estimation exploits both the likelihood of the reconstructed EMG signal and some physiological constraints, such as the discharge pattern regularity and the refractory period of muscle fibers, as prior information integrated in a Bayesian framework. A Tabu search is proposed to efficiently tackle the nondeterministic polynomial-time-hard problem of optimization w.r.t the motor unit discharge patterns. The method is fully automatic and was tested on simulated and experimental EMG signals. Compared with the semiautomatic decomposition performed by an expert operator, the proposed method resulted in an accuracy of $\mathbf{9 0 . 0 \%} \pm \mathbf{3 . 8 \%}$ when decomposing singlechannel intramuscular EMG signals recorded from the abductor digiti minimi muscle at contraction forces of $5 \%$ and $10 \%$ of the maximal force. The method can also be applied to the automatic identification and classification of spikes from other neural recordings.
\end{abstract}

Index Terms-Bayesian analysis, electromyography (EMG) signal decomposition, Tabu search.

\section{INTRODUCTION}

I NTRAMUSCULAR electromyography (EMG) signals are the sum of series of action potentials discharged by the motor units (MUs) within the detection volume of the recording electrodes. The interference intramuscular EMG signals can be decomposed into the constituent MU action potentials (MUAPs) to extract the discharge patterns of the motor neurons innervating the muscle. This procedure allows the analysis of MU control properties [1] and thus provides a unique insight into the neural drive to muscles. EMG signal decomposition is usually performed by semiautomated methods, often based on serial detection and classification of action potentials.

Manuscript received February 3, 2009; revised April 9, 2009. First published May 19, 2009; current version published February 17, 2010. Asterisk indicates corresponding author.

D. Ge and E. Le Carpentier are with the Institut de Recherche en Communications et Cybernétique de Nantes, Unité Mixte de Recherche 6597, Centre National de la Recherche Scientifique, École Centrale de Nantes, 44321 Nantes Cedex 03, France (e-mail: di.ge@irccyn.ec-nantes.fr; eric.lecarpentier@irccyn.ec-nantes.fr).

${ }^{*} \mathrm{D}$. Farina is with the Department of Health Science and Technology, Center for Sensory-Motor Interaction, Aalborg University, Aalborg DK-9220, Denmark (e-mail: df@hst.aau.dk).

Color versions of one or more of the figures in this paper are available online at http://ieeexplore.ieee.org.

Digital Object Identifier 10.1109/TBME.2009.2022277
Since the pioneer work completed by De Luca and coworkers both in the development of detection needles, processing techniques and in the use of EMG signal decomposition for understanding fundamental physiological issues [1]-[4], many techniques have been further developed for the EMG decomposition (for review, see [5]). In classical approaches for intramuscular EMG decomposition, the MUAPs are identified from the interference signal with a threshold and classified as belonging to the active MUs. The challenge in the decomposition is mainly the separation of MUAPs that overlap in time. The global optimization of overlapped MUAPs is indeed an nondeterministic polynomial-time (NP) hard problem, i.e., a problem that cannot be solved by polynomial complexity algorithms. The existing methods either perform on the restrained search spaces [6], [7] to reduce the complexity or are based on recursive algorithms [8], [9] with specific trial strategies and residual threshold estimations. Atiya [7] proposed a robust approach for decomposing overlaps of action potentials in neural recordings by comparing all possible combinations of up to two action potentials (restrained search space). Within the family of the spike sorting algorithms, the dynamic programming method [6], which uses the fast exploration technique of a $\mathrm{k}-\mathrm{d}$ tree, is also limited by the memory space necessary to generate such data structure, resulting in an equally restrained search space of up to two overlapping action potentials. This restraint is not justified in several applications, for example, when decomposing intramuscular EMG signals comprising many active MUs. Other approaches are not limited in the number of overlapping sources, however, they usually require the interaction with an operator. For example, the EMGLAB algorithm [8], [9] recursively matches the templates (reference MUAPs) and reevaluates the residual errors, similar to the greedy algorithm, but requires an expert intervention when the algorithm fails to reach the residual lower bound [8] after a number of trials.

In this study, we propose a fully automatic EMG decomposition algorithm that exploits both the signal model likelihood and the regularity of the MU discharge patterns in a similar way as it is done during the manual decomposition. Unlike previous methods, which indirectly use the information on MU discharge regularity in an interactive procedure involving human intervention [9], the proposed method integrates this information as prior law in a Bayesian framework. The NP-hard problem associated to the decomposition of overlapping MUAPs is solved with the Tabu metaheuristic without limiting the search space. Moreover, contrary to methods for the multichannel surface EMG decomposition [10]-[12], the proposed method requires one single recording channel. The method was tested on simulated and experimental signals. 


\section{MODEL AND METHOD}

\section{A. Forward Model}

An interference EMG signal comprising the contributions of $I$ MU discharge sources can be described as

$$
\boldsymbol{z}=\sum_{i=1}^{I} \boldsymbol{h}_{i} * \boldsymbol{s}_{i}+\epsilon
$$

where $z$ is the recorded EMG signal of length $N$, modeled as a mixture of convolutions of the impulse trains $\boldsymbol{s}_{i}, i=1, \ldots, I$ (the discharge patterns) and the linear filters $\boldsymbol{h}_{i}, i=1, \ldots, I$ (MUAPs). The shorthand $(\bullet)_{i}$ replaces the whole set $\{\bullet, i=$ $1 \ldots, I\}$ without ambiguity, thus $\left(\boldsymbol{s}_{i}\right)_{i}=\left\{\boldsymbol{s}_{i}, i=1 \ldots, I\right\}$. The following statistical assumptions are made on this mixture of sources.

1) Each impulse source $\boldsymbol{s}_{i}=1_{\boldsymbol{x}_{i}}$ (the discharge pattern) is modeled as an arriving-time process with uniform amplitudes. The subscript of the indicator function $\boldsymbol{x}_{i}$ is a vector containing the impulse coordinates for the $i$ th source (arriving time of impulses). We also denote $n_{i}=\operatorname{dim}\left(\boldsymbol{x}_{i}\right)$, the number of discharges.

2) The MU discharge patterns $\left(1_{\boldsymbol{x}_{i}}\right)_{i}$ are supposed mutually independent.

3) The MUAP shapes $\left(\boldsymbol{h}_{i}\right)_{i}$ vary slowly during the EMG recording (i.e. variations in shape of the MUAPs occur during time intervals of seconds as order of magnitude).

4) The observation $z$ is corrupted by an additive white Gaussian noise with unknown variance $\sigma_{\epsilon}^{2}$.

In relation to assumption 2 ), it should be noted that two types of correlations exist between MU discharge patterns. The first consists of the common modulation of the time-varying discharge rates between pairs of MUs (common drive) [1]. The second is characterized by simultaneous or near-simultaneous discharges of two or more MUs (including but not limited to phase locked and entrained $\left.\left(1_{\boldsymbol{x}_{i}}\right)_{i}\right)$ that occur more often than would be expected with independent processes (short-time synchronization [13], [14]). Models exist (e.g., see [15]) to simulate the interdependent discharge patterns, by introducing both MU common drive and short-time synchronization among MUs. However, these two types of correlation between MU discharge patterns cannot be directly coded into the prior laws of $\left(1_{\boldsymbol{x}_{i}}\right)_{i}$. This a priori information is thus ignored in the present study to yield a simple model structure. It is also as a general rule in Bayesian inference problems that independency assumptions often imply lack of tractability to exploit the data dependency properties rather than proved scientific observations.

The data-generating process, given the source parameters $\left(\boldsymbol{x}_{i}, \boldsymbol{h}_{i}\right)_{i}, \sigma_{\epsilon}^{2}$, obeys the Gaussian law

$$
\begin{aligned}
& P\left(\boldsymbol{z} \mid\left(\boldsymbol{x}_{i}, \boldsymbol{h}_{i}\right)_{i}, \sigma_{\epsilon}^{2}\right) \\
& \quad=\left(\frac{1}{\sqrt{2 \pi \sigma_{\epsilon}^{2}}}\right)^{N} \exp \left(-\frac{\left\|\boldsymbol{z}-\sum_{i} 1_{\boldsymbol{x}_{i}} * \boldsymbol{h}_{i}\right\|^{2}}{2 \sigma_{\epsilon}^{2}}\right) .
\end{aligned}
$$

The following additional assumptions are made on the discharge pattern of each MU.
1) The interspike interval (ISI) $T_{i j}=\boldsymbol{x}_{i, j+1}-\boldsymbol{x}_{i, j}$ between two consecutive impulses for a given source $s_{i}$ is larger than a threshold value $T_{R}$, which indicates a physiological constraint on the ISI (e.g., $T_{R}$ may be set similar to the refractory period of muscle fibers).

2) $\left(T_{i j}-T_{R}\right)$ follows a Gaussian-shaped distribution $\left(T_{i j}-\right.$ $\left.T_{R}\right) \sim \mathcal{N}\left(m_{i}, \sigma_{i}^{2}\right)$

3) The ISI variability is smaller than a threshold: $\sigma_{i} / m_{i}<$ $\mathrm{Th}_{\sigma_{i}}$; this constraint expresses the regularity of the discharge patterns and the threshold $\mathrm{Th}_{\sigma_{i}}$ controls the variability of the ISI.

According to the previous assumptions, the ISI follows a truncated Gaussian distribution, in which

$$
\begin{cases}P\left(T_{i j}\right)=0, & T_{i j}<T_{R} \\ P\left(T_{i j}-T_{R}\right) \propto g\left(m_{i}, \sigma_{i}^{2}\right), & T_{i j} \geq T_{R}\end{cases}
$$

This assumption is in accordance with the histograms of experimentally observed discharge patterns [16], i.e. approximately Gaussian-shaped with a normalized variance $\sigma_{i} / m_{i}<\mathrm{Th}_{\sigma_{i}}$. On a discrete grid, the ISI probability in (3) is well-defined up to a normalization factor. The source-generating process is thus the following:

$$
\begin{aligned}
& P\left(\boldsymbol{x}_{i} \mid m_{i}, \sigma_{i}^{2}\right) \\
& =\frac{1}{4} \operatorname{erfc}\left(\frac{x_{i, 1}-T_{R}-m_{i}}{\sqrt{2} \sigma_{i}}\right) \operatorname{erfc}\left(\frac{N-x_{i, n_{i}}-T_{R}-m_{i}}{\sqrt{2} \sigma_{i}}\right) \\
& \left(\frac{1}{2 \pi \sigma_{i}^{2}}\right)^{\left(n_{i}-1\right) / 2} \exp \left(-\sum_{j=1}^{n_{i}-1} \frac{\left(\boldsymbol{x}_{i, j+1}-\boldsymbol{x}_{i, j}-m_{i}-T_{R}\right)^{2}}{2 \sigma_{i}^{2}}\right)
\end{aligned}
$$

for a given configuration of a source $\boldsymbol{s}_{i}=1_{\boldsymbol{x}_{i}}$. The two terms $1 / 2 \operatorname{erfc}(\bullet)$ in (4) evaluate the first impulse ISI with probability $P\left(T_{i, 1}>x_{i, 1}\right)$ and the $\left(n_{i}+1\right)^{(\text {th })}$ impulse ISI with probability $P\left(T_{i, n_{i}+1}>N-x_{i, n_{i}}\right)$ respectively. For simplicity, the endeffects by integration are neglected in the following, so that we obtain

$$
\begin{aligned}
P\left(\boldsymbol{x}_{i} \mid m_{i}, \sigma_{i}^{2}\right) \approx & \left(\frac{1}{2 \pi \sigma_{i}^{2}}\right)^{\left(n_{i}-1\right) / 2} \\
& \times \exp \left(-\sum_{j=1}^{n_{i}-1} \frac{\left(\boldsymbol{x}_{i, j+1}-\boldsymbol{x}_{i, j}-m_{i}-T_{R}\right)^{2}}{2 \sigma_{i}^{2}}\right) .
\end{aligned}
$$

With the independence assumption of MU discharge patterns $\left(\boldsymbol{x}_{i}\right)_{i}$, we also obtain

$$
P\left(\left(\boldsymbol{x}_{i}\right)_{i} \mid\left(m_{i}, \sigma_{i}^{2}\right)_{i}\right)=\prod_{i=1}^{I} P\left(\boldsymbol{x}_{i} \mid m_{i}, \sigma_{i}^{2}\right)
$$

In the following, $P\left(\left(\boldsymbol{x}_{i}\right)_{i}\right)$ will denote the prior law for the discharge patterns to simplify the notation. 


\section{B. Posterior Probability Law}

In a Bayesian framework, we establish the posterior distribution for the unknown variables $\left\{\left(\boldsymbol{x}_{i}, m_{i}, \sigma_{i}^{2}, \boldsymbol{h}_{i}\right)_{i}, \sigma_{\epsilon}^{2} \mid \boldsymbol{z}\right\}$ :

$$
\begin{aligned}
P\left(\left(\boldsymbol{x}_{i}, m_{i}, \sigma_{i}^{2}, \boldsymbol{h}_{i}\right)_{i}, \sigma_{\epsilon}^{2} \mid \boldsymbol{z}\right) \propto P\left(\boldsymbol{z} \mid\left(\boldsymbol{x}_{i}, \boldsymbol{h}_{i}\right)_{i}, \sigma_{\epsilon}^{2}\right) & \\
& \prod_{i=1}^{I}\left(P\left(\boldsymbol{x}_{i} \mid m_{i}, \sigma_{i}^{2}\right) P\left(m_{i}\right) P\left(\sigma_{i}^{2}\right) P\left(\boldsymbol{h}_{i}\right)\right) P\left(\sigma_{\epsilon}^{2}\right) .
\end{aligned}
$$

The decomposition task is accomplished by jointly maximizing (7) w.r.t. the discharge patterns $\left(\boldsymbol{x}_{i}\right)_{i}$, their statistics $\left(m_{i}, \sigma_{i}^{2}\right)_{i}$, the MUAP shapes $\left(\boldsymbol{h}_{i}\right)_{i}$ and the background noise variance $\sigma_{\epsilon}^{2}$. Equations (2) and (4) provide the first two terms of the righthand side of (7). The remaining terms can be expressed as conjugate priors with noninformative hyperparameters $\left(\alpha_{i}, \beta_{i}\right)_{i}$, $\left(\alpha_{s}, \beta_{s}, \mu_{0}, \sigma_{0}^{2}, \sigma_{\boldsymbol{h}}^{2}\right)$

$$
\begin{aligned}
P\left(m_{i}\right) & \sim \mathcal{N}\left(\mu_{0}, \sigma_{0}^{2}\right), & P\left(\sigma_{i}^{2}\right) & \sim \mathcal{I} \mathcal{G}\left(\alpha_{i}, \beta_{i}\right) \\
P\left(\boldsymbol{h}_{i}\right) & \sim \mathcal{N}\left(\boldsymbol{h}_{i}^{(0)}, \sigma_{\boldsymbol{h}}^{2}\right), & P\left(\sigma_{\epsilon}^{2}\right) & \sim \mathcal{I} \mathcal{G}\left(\alpha_{s}, \beta_{s}\right)
\end{aligned}
$$

where $\mathcal{I} \mathcal{G}$ stands for the inverse Gamma distribution.

For example, the inverse Gamma $\mathcal{I} \mathcal{G}(1,1)$ yields an almost uniform distribution that covers a wide range of parameter values. The choice of $\mu_{0}, \sigma_{0}^{2}$ expresses the prior information on the discharge rate of each MU and the certainty of this information. Typically, the Gaussian prior of $m_{i}$ can be interpreted as conveying no specific prior information under the condition that $[25 \mathrm{~ms}$, $200 \mathrm{~ms}] \subset\left[\mu_{0}-3 \sigma_{0}, \mu_{0}+3 \sigma_{0}\right]$ (i.e. most of the physiological discharge rates ranging from 5 to $40 \mathrm{~Hz}$ are considered, corresponding to a mean value of 200 and $25 \mathrm{~ms}$, respectively). The same remark is applied to $\sigma_{\boldsymbol{h}}^{2}$ that controls the uncertainty of the initialized shapes $\left(\boldsymbol{h}_{i}^{(0)}\right)_{i}$, which are the approximated MUAP obtained from the preprocessing phase (see Section III-A).

\section{MAXIMIZATION AlgORITHM}

This section describes the main features of the two-phase EMG signal decomposition algorithm based on the maximum a posteriori estimator (MAP) applied to the forward model described earlier; the joint posterior distribution of (7) is maximized w.r.t. the unknown parameters

$$
\widehat{\Theta}=\arg \max P(\Theta \mid \boldsymbol{z})
$$

with $\Theta=\left\{\left(\boldsymbol{x}_{i}, \boldsymbol{h}_{i}, m_{i}, \sigma_{i}^{2}\right)_{i}, \sigma_{\epsilon}^{2}\right\}$.

The Section III-A presents the overall structure of the method, divided into a preprocessing phase and a complete EMG separation phase that iteratively maximizes (7). For fixed $\left(\boldsymbol{x}_{i}\right)_{i}$, maximization w.r.t. $\left\{\left(\boldsymbol{h}_{i}, m_{i}, \sigma_{i}^{2}\right)_{i}, \sigma_{\epsilon}^{2}\right\}$ leads to a closed-form solution. On the other hand, the problem of estimating the discharge pattern $\left(\boldsymbol{x}_{i}\right)_{i}$ cannot be practically solved by an exhaustive exploration due to computational time [6], [17]. The decomposition problem consists of determining the MU label set and the discharge instants that maximize the posterior distribution expressed by (7). The complete search space of the discharge pattern $\left(\boldsymbol{x}_{i}\right)_{i}$, containing all possible alignments of all possible combinations of MU label sets, is exponential [18]. In this paper, we propose to use the Tabu search algorithm [19] to deal with the maximization w.r.t. the discharge pattern $\left(\boldsymbol{x}_{i}\right)_{i}$.
We clarify the main application issues such as the cost function to minimize (equivalent to the maximization of the marginal posterior law for $\left(\boldsymbol{x}_{i}\right)_{i}$ ), the neighborhood definition (the set of moves allowed in one iteration), and the Tabu list structure.

\section{A. Overall Structure}

The decomposition method consists of two phases. First, the EMG signal is segmented and a few representatives of the MUAPs $\left(\boldsymbol{h}_{i}^{(0)}\right)_{i}$ are extracted for initialization of MUAP shapes [see (9)]. It is implemented as a classic preprocessing for MUAP detection in intramuscular recordings and comprises bandpass filtering, thresholding for detection [17], [18], and isolated MUAP shape classification [20]. In this study, we applied a level threshold proportional to the background noise standard deviation estimate $\widehat{\sigma_{\epsilon}}$. The active segments $\left(\left\{\operatorname{Seg}_{k}\right\}\right)$ are then detected where either isolated or overlapped MUAPs occur. False positives are preferred over false negatives since $\left(\boldsymbol{x}_{i}\right)_{i}=\phi$ in one active segment is an allowed solution of the MAP estimation (the second phase).

The algorithms used in the first phase, inspired from the methods in [9], [20], are as follows.

1) Bandpass filtering of the raw EMG signal to enhance the MUAP's fast rising edges while suppressing the low-frequency background activities. The first-order differentiator in [20] is adopted for its simplicity, as in [9]: $\boldsymbol{z}_{n}^{f}=\boldsymbol{z}_{n+1}-\boldsymbol{z}_{n-1}$, where $z$ and $z^{f}$ represent the raw EMG data and its filtered output, respectively. In the following, $z \leftarrow \boldsymbol{z}^{f}$ while we keep the notation $z$ for filtered EMG.

2) Segmenting the prefiltered EMG signal into temporal intervals that contain either overlapped or separated MUAP activities. A level threshold $\mathrm{Th}_{\mathrm{Seg}}$ is calculated based on a quick estimation of $\sigma_{\epsilon}$ by solving the implicit equation

$$
\mathrm{Th}_{\text {Seg }}=4 \sqrt{\frac{\sum_{n=1}^{T_{1}} \boldsymbol{z}_{n}^{2} I\left(\mathrm{Th}_{\mathrm{Seg}}, n\right)}{\sum_{n=1}^{T_{1}} I\left(\mathrm{Th}_{\mathrm{Seg}}, n\right)}}
$$

in which the indicator function $I(p, n)=1$ if $\left|\boldsymbol{z}_{n}\right|<p$ and 0 otherwise. We fix $T_{1}$ as the first 1 -s interval of $\boldsymbol{z}$, i.e. 10000 samples if the sampling frequency is $10 \mathrm{kHz}$ and the coefficient 4 in (10) corresponds to detect only MUAP activities whose absolute amplitudes exceed $4 \sigma_{\epsilon}$. The solution of (10) is of complexity $\mathcal{O}\left(T_{1}\right)$ (code given in EMGLAB [9]). We then detect all the local extrema of $z$ whose absolute values exceed $\mathrm{Th}_{\mathrm{Seg}}$ (empirically believed to be MUAP peaks). A new segment is allocated when the distance between two adjacent extrema exceeds $2 P+1, P$ being the length of $\left(\boldsymbol{h}_{i}\right)_{i}$. An example of two segments is provided in Fig. 2 for which $P$ is set to $5 \mathrm{~ms}$.

3) Extracting recurrent isolated MUAP forms from the segments to obtain $\left(\boldsymbol{h}_{i}^{(0)}\right)_{i}$. Canonically registered discrete Fourier transform (CRDFT) coefficients (see [20] and [21], and references therein for implementation details) are calculated on segments with lengths smaller than $3 P$ to exclude those potentially containing overlapped waveforms and a quadratic metric is adopted on these CRDFT coefficients to make a one-pass classification operation. The progressively averaged CRDFT 
coefficients are considered as the DFT of the representative templates of $\left(\boldsymbol{h}_{i}^{(0)}\right)_{i}$, used as initialization in the second phase.

The segment length depends on the signal properties and the parameters used for segmentation, such as the sparsity of $\boldsymbol{z}$ (basically depending on the number of active MUs) and the chosen MUAP length $P$. Since the optimization of (7) w.r.t. $\left(1_{\boldsymbol{x}_{i}}\right)_{i}$ is only performed for intervals of the active segments, the segmentation step reduces the computational burden of the second phase. The first phase of the decomposition, as described earlier, is completely automated and requires a negligible computational load compared to the second phase, that solves the problem of MUAP overlaps.

The novel part of the decomposition method consists of the second phase. After segmentation, the maximizationdecomposition phase is applied serially to each active segment $\left\{\operatorname{Seg}_{k}\right\}$ to obtain: 1) the complete discharge patterns $\left(\boldsymbol{x}_{i}\right)_{i}$ for each MUAP; 2) the MUAP shape estimates $\left(\boldsymbol{h}_{i}\right)_{i}$; and 3) the background noise variance $\sigma_{\epsilon}^{2}$, with an MAP criterion. For each segment, the joint posterior distribution [see (7)] is maximized over the complete search space of the discharge patterns $\left(\boldsymbol{x}_{i}\right)_{i}$ in an automatic algorithm. The kernel of this problem (MUAP overlapping) is dealt with by a metaheuristic algorithm, called Tabu search, first introduced by Glover [19] in the area of operational research. The generic Tabu search algorithm and its application are described in Section III-C. The optimization strategy is designed to overcome limitations such as early convergence into local optima in NP-hard problems and is thus adapted to solve the combinatorial problem of MUAP overlaps.

The MAP optimization are summarized as follows.

1) Initialize $\left(\boldsymbol{h}_{i}^{(0)}\right)_{i}$ (MUAP shapes) using the information from phase 1 (segmentation).

2) Initialize $\left(m_{i}, \sigma_{i}^{2}\right)_{i}$ by their conjugate prior laws, and $\sigma_{\epsilon}$ by $\widehat{\sigma}_{\epsilon}$ from $(10)$.

3) Initialize $\boldsymbol{x}_{i}=\phi\left(1_{\boldsymbol{x}_{i}}=\mathbf{0}\right)$ for all $i$.

4) Iterate:

a) For each $\mathrm{Seg}_{k}$, maximize:

$$
P\left(\left(\boldsymbol{x}_{i}^{(k)}\right)_{i} \mid\left(\boldsymbol{x}_{i}^{(-k)}, \boldsymbol{h}_{i}, m_{i}, \sigma_{i}^{2}\right)_{i}, \sigma_{\epsilon}^{2}, \boldsymbol{z}\right)
$$

w.r.t. $\left(\boldsymbol{x}_{i}^{(k)}\right)_{i}$ using Tabu search

b) Maximize (7) w.r.t. continuous parameters $\left(\boldsymbol{h}_{i}, m_{i}, \sigma_{i}^{2}\right)_{i}, \sigma_{\epsilon}^{2}$ (see Section III-B);

until convergence.

In the previous steps, $\boldsymbol{x}_{i}^{(k)}=\boldsymbol{x}_{i} \cap \mathrm{Seg}_{k}, i=1, \ldots, I$, denote the discharge coordinates vector of each MU within the given $\mathrm{Seg}_{k}$ and the superscript $(-k)$ in the following indicates variables belonging to segments $\cup_{j \neq k} \operatorname{Seg}_{j}$.

The calculations in the step 4(b) are straightforward maximization problems, for which we provide detailed expressions in Section III-B. Step 4(a), however, is a typical NP-hard combinatorial problem whose complete space amounts to $2^{I \mathrm{dim}\left(\mathrm{Seg}_{k}\right)}$ configurations for the $k$ th active segment, where $I$ denotes the number of classes (MUs) and $\operatorname{dim}\left(\mathrm{Seg}_{k}\right)$ the length of the segment. Its maximization algorithm is explained in Section III-C using the Tabu search metaheuristic. Step 4(a) is solved by an iterative Tabu search that runs until convergence is detected either by exhausting all valid neighbors or by reaching the max- imum allowed iteration counts $M$ as stated in the algorithm in Section III-C. The external loop is run for both the steps 4(a) and (b) until the global convergence of $\Theta$. Jointly, the steps 4(a) and (b) bear a certain resemblance to a Gibbs simulation scheme [22]. However, while a Gibbs simulation scheme iteratively generates samples asymptotically distributed to (7), the proposed algorithm iteratively maximizes the posterior probability in (7), and finally, builds an estimate of $\Theta$ in an MAP sense.

\section{B. Maximization on Continuous Parameters}

We describe first the maximization procedures in step 4(b).

1) Maximization w.r.t. $m_{i}$ : The maximization problem can be written as: for all $i$,

$$
\begin{aligned}
m_{i} & =\max _{m_{i}} P(\Theta \mid \boldsymbol{z})=\max _{m_{i}} P\left(\boldsymbol{x}_{i} \mid m_{i}, \sigma_{i}^{2}\right) P\left(m_{i}\right) \\
& =\max _{m_{i}}\left(\exp \left\{-\frac{1}{2 \sigma_{i}^{2}} \sum_{j=1}^{n_{i}-1}\left(S_{i j}-m_{i}\right)^{2}\right\} P\left(m_{i}\right)\right)
\end{aligned}
$$

where $\left\{S_{i j}=\boldsymbol{x}_{i, j+1}-\boldsymbol{x}_{i, j}-T_{R}\right\}$ is the set of Gaussian samples. $P\left(\boldsymbol{x}_{i} \mid m_{i}, \sigma_{i}^{2}\right)$ is given in (5). Since its prior law $P\left(m_{i}\right)$ is also a Gaussian and the product of two Gaussian is still Gaussian, the maximum is found at

$$
m_{i}=\frac{\left(\mu_{0} / \sigma_{0}^{2}+\left(\sum_{j=1}^{n_{i}-1} S_{i j}\right) / \sigma_{i}^{2}\right)}{\left(1 / \sigma_{0}^{2}+\left(n_{i}-1\right) / \sigma_{i}^{2}\right)} .
$$

2) Maximization w.r.t. $\sigma_{i}^{2}$ : The maximization problem can be written as: for all $i$,

$$
\begin{aligned}
\sigma_{i}^{2} & =\max _{\sigma_{i}^{2}} P(\Theta \mid \boldsymbol{z})=\max _{\sigma_{i}^{2}} P\left(\boldsymbol{x}_{i} \mid m_{i}, \sigma_{i}^{2}\right) P\left(\sigma_{i}^{2}\right) \\
& =\max _{\sigma_{i}^{2}} \sigma_{i}^{-\left(n_{i}-1\right)} \exp \left\{-\frac{1}{2 \sigma_{i}^{2}} \sum_{j=1}^{n_{i}-1}\left(S_{i j}-m_{i}\right)^{2}\right\} P\left(\sigma_{i}^{2}\right) .
\end{aligned}
$$

Since the inverse Gamma distribution is the conjugate prior for Gaussian likelihood with known mean, their product is still inverse Gamma with the maximum found at

$$
\sigma_{i}^{2}=\frac{\beta_{i}+\left(\sum_{j=1}^{n_{i}-1}\left(S_{i j}-m_{i}\right)^{2}\right) / 2}{\alpha_{i}+1+\left(n_{i}-1\right) / 2} .
$$

To take into account the regularity constraint that $\sigma_{i} / m_{i}<$ $\mathrm{Th}_{\sigma_{i}}$, we impose that $\sigma_{i}^{2} \leftarrow \min \left\{\sigma_{i}^{2},\left(\mathrm{Th}_{\sigma_{i}} m_{i}\right)^{2}\right\}$. This constraint can be tuned depending on the expected regularity of the discharge pattern.

3) Maximization w.r.t. $\left(\boldsymbol{h}_{i}\right)_{i}$ : We propose to jointly maximize $\left(\boldsymbol{h}_{i}\right)_{i}$ by creating a concatenated column vector of size $I P \times 1: \boldsymbol{h}=\left[\boldsymbol{h}_{1}, \ldots, \boldsymbol{h}_{I}\right]$ and the maximization problem can be written as

$$
\begin{aligned}
\boldsymbol{h} & =\max _{\boldsymbol{h}} P(\Theta \mid \boldsymbol{z})=\max _{\boldsymbol{h}} P\left(\boldsymbol{z} \mid\left(\boldsymbol{x}_{i}\right)_{i}, \boldsymbol{h}, \sigma_{\epsilon}^{2}\right) P(\boldsymbol{h}) \\
& =\max _{\boldsymbol{h}}\left(\exp \left\{-\frac{1}{2 \sigma_{\epsilon}^{2}}\|\boldsymbol{z}-\mathbf{M} \boldsymbol{h}\|^{2}\right\} P(\boldsymbol{h})\right)
\end{aligned}
$$

with $\mathbf{M}=\left[\mathbf{M}_{1}, \ldots, \mathbf{M}_{I}\right]$, where each submatrix $\mathbf{M}_{i}$ is the Toeplitz matrix of convolution such that $\mathbf{M}_{i} \boldsymbol{h}_{i}=1_{\boldsymbol{x}_{i}} * \boldsymbol{h}_{i}$. 
It follows that $\boldsymbol{z}-\mathbf{M} \boldsymbol{h}=\boldsymbol{z}-\sum_{i} 1_{\boldsymbol{x}_{i}} * \boldsymbol{h}_{i}$. Since $P(\boldsymbol{h}) \sim$ $\mathcal{N}\left(\boldsymbol{h}^{(0)}, \sigma_{h}^{2} \boldsymbol{I}\right)$, the maximum of the marginal posterior distribution is found at

$$
\boldsymbol{h}=\left(\sigma_{\boldsymbol{h}}^{-2} \boldsymbol{I}+\sigma_{\epsilon}^{-2} \mathbf{M}^{t} \mathbf{M}\right)^{-1}\left(\sigma_{\boldsymbol{h}}^{-2} \boldsymbol{h}^{(0)}+\sigma_{\epsilon}^{-2} \mathbf{M}^{t} \boldsymbol{z}\right) .
$$

We note that the matrix $\sigma_{\boldsymbol{h}}^{-2} \boldsymbol{I}+\sigma_{\epsilon}^{-2} \mathbf{M}^{t} \mathbf{M}$ is $P I \times P I$ positive definite; thus, its inversion operation can be replaced by a Cholesky factorization followed by matrix left division to reduce the calculation load. A minimum degree preordering can also be used since $\mathbf{M}^{\mathrm{t}} \mathbf{M}$ is sparse for any MU source. The motivation behind the joint maximization of $\left(\boldsymbol{h}_{i}\right)_{i}$ is to ensure one-step convergence to the joint Gaussian mode, instead of a coordinate-by-coordinate maximization.

4) Maximization w.r.t. $\sigma_{\epsilon}^{2}$ : The maximization problem can be written as follows:

$$
\begin{aligned}
\sigma_{\epsilon}^{2} & =\max _{\sigma_{\epsilon}^{2}} P(\Theta \mid \boldsymbol{z})=\max _{\sigma_{\epsilon}^{2}} P\left(\boldsymbol{z} \mid\left(\boldsymbol{x}_{i}, \boldsymbol{h}_{i}\right)_{i}, \sigma_{\epsilon}^{2}\right) P\left(\sigma_{\epsilon}^{2}\right) \\
& =\max _{\sigma_{\epsilon}^{2}} \sigma_{\epsilon}^{-N} \exp \left\{-\frac{1}{2 \sigma_{\epsilon}^{2}} \mathbf{C}\right\} P\left(\sigma_{\epsilon}^{2}\right)
\end{aligned}
$$

where $\mathbf{C}=\|\boldsymbol{z}-\mathbf{M} \boldsymbol{h}\|^{2}$ denotes the energy of the reconstruction error. And the maximum is found at

$$
\sigma_{\epsilon}^{2}=\frac{\beta_{s}+\mathbf{C} / 2}{\alpha_{s}+1+(N / 2)} .
$$

\section{Maximization on Combinatorial Sets}

A Tabu search approach [19] is applied to solve step 4(a) as the following optimization problem:

$$
\text { Minimize: } c(y) \in \mathcal{R}: y \in Y \text {. }
$$

The objective function $c(y)$ should be a real-valued cost function, while $Y$ is a discrete set containing all combinations of $y$. Associated with each $y \in Y$ is the set Neighbor $(y)$ composed of all possible moves (trial solutions) from a current configuration $y$. A subset $T \subset Y$, the Tabu list, comprising prohibited moves is managed using a first-in-first-out (FIFO) structure. All allowed trials from a given state $y$ belongs to the set Neighbor $(y) \backslash T$. The Tabu search tackles the optimization problem in (15) as follows:



First, the nonimproving moves are made possible through step 4 since we do not necessarily have $c\left(y_{m}\right) \leq c\left(y_{m-1}\right)$ : a best move rather than an improving move is chosen. Second, visited local optima are forbidden for future moves by the FIFO mechanism of $T$ that remembers to a certain degree (depending on the FIFO length) local optima to avoid. We begin by reformulating the step 4(a) from an optimization point of view. Let $n_{i}^{(k)}=\operatorname{dim}\left(\boldsymbol{x}_{i}^{(k)}\right)$ denote the discharge counts of MU $i$ in $\operatorname{Seg}_{k}$. It follows that

$$
\begin{aligned}
& \underset{\left(\boldsymbol{x}_{i}\right)_{i}^{(k)}}{\arg \max } P\left(\left(\boldsymbol{x}_{i}\right)_{i}^{(k)} \mid\left(\boldsymbol{x}_{i}^{(-k)}, \boldsymbol{h}_{i}, m_{i}, \sigma_{i}^{2}\right)_{i}, \sigma_{\epsilon}^{2}, \boldsymbol{z}\right) \\
& \underset{\left(\boldsymbol{x}_{i}\right)_{i}^{(k)}}{\arg \max \exp }\left\{\frac{-\left\|\boldsymbol{z}^{(k)}-\sum_{i=1}^{I} \boldsymbol{h}_{i} * 1_{\boldsymbol{x}_{i}}^{(k)}\right\|^{2}}{\left(2 \sigma_{\epsilon}^{2}\right)}\right\} \\
& \prod_{i=1}^{I} \sigma_{i}^{-n_{i}^{(k)}} \exp \left(-\sum_{j=0}^{n_{i}^{(k)}} \frac{\left(\boldsymbol{x}_{i, j+1}^{(k)}-\boldsymbol{x}_{i, j}^{(k)}-m_{i}-T_{R}\right)^{2}}{2 \sigma_{i}^{2}}\right) \\
& =\underset{\left(\boldsymbol{x}_{i}\right)_{i}^{(k)}}{\arg \min } \frac{1}{2 \sigma_{\epsilon}^{2}}\left\|\boldsymbol{z}^{(k)}-\sum_{i=1}^{I} \boldsymbol{h}_{i} * 1_{\boldsymbol{x}_{i}}^{(k)}\right\|^{2}+\log \sigma_{i} \sum_{i}^{I} n_{i}^{(k)} \\
& \quad+\sum_{i}^{I}\left(\sum_{j=0}^{n_{i}^{(k)}} \frac{\left(\boldsymbol{x}_{i, j+1}^{(k)}-\boldsymbol{x}_{i, j}^{(k)}-m_{i}-T_{R}\right)^{2}}{2 \sigma_{i}^{2}}\right)
\end{aligned}
$$

where for MU $i, \boldsymbol{x}_{i, 0}^{(k)}$ and $\boldsymbol{x}_{i, n_{i}^{(k)}+1}^{(k)}$ denote the last discharge in the $(k-1)$ th segment and the first discharge in the $(k+$ 1)th segment, respectively. The first term in the criterion of (16) can be identified as the likelihood of the EMG signal $z$ given $\left(\boldsymbol{h}_{i}, 1_{\boldsymbol{x}_{i}}\right)_{i}$ and the last two terms as the prior information (regularity) of the discharge patterns. From the Bayesian point of view, they are naturally combined through the marginal posterior distribution on $\left(\boldsymbol{x}_{i}\right)_{i}$ in the equation.

In the following, the superscript $(k)$ is omitted without ambiguity since only $\left(\boldsymbol{x}_{i}\right)_{i}^{(k)}$ is considered for each segment. The following elements are defined to apply the Tabu search:

1) $y=\left(\boldsymbol{x}_{i}\right)_{i}$ and $Y=\left(\{0,1\}^{\operatorname{dim}\left(\operatorname{Seg}_{k}\right)}\right)^{I}$ define a state and the search space respectively;

2) the cost function $c(y)$ is expressed in (16);

3) a cyclic fixed-length $T$ (Tabu list) is updated to keep track of previous solutions; each state is characterized by MUAP classes and discharge instants;

4) for a given state, Neighbor $\left(\left(\boldsymbol{x}_{i}\right)_{i}\right)$ is defined as:

$$
\left\{\left(\boldsymbol{x}_{i}^{*}\right)_{i}\left|\sum_{i}\right| 1_{\boldsymbol{x}_{i}^{*}}-\left.1_{\boldsymbol{x}_{i}}\right|_{1} \leq 2, \sum_{i}\left|n_{i}^{*}-n_{i}\right|<2\right\}
$$

in which $|\cdot|_{1}$ defines the L1-norm of the vector (or the Hamming distance in the case of two binary sequences).

It can be verified that such a neighborhood allows either adding a spike at any unoccupied position of the same MUAP, removing or changing the position of an existent spike. Furthermore, the neighborhood is both selfincluded $\left(\left(\boldsymbol{x}_{i}\right)_{i} \in \operatorname{Neighbor}\left(\left(\boldsymbol{x}_{i}\right)_{i}\right)\right)$ and reflective $\left(\left(\boldsymbol{x}_{i}\right)_{i}^{*} \in\right.$ Neighbor $\left.\left(\left(\boldsymbol{x}_{i}\right)_{i}\right) \rightleftharpoons\left(\boldsymbol{x}_{i}\right)_{i} \in \operatorname{Neighbor}\left(\left(\boldsymbol{x}_{i}\right)_{i}^{*}\right)\right)$. It is also easily verified that the cardinality of Neighbor $\left(\left(\boldsymbol{x}_{i}\right)_{i}\right)$ amounts to $\left(I+\sum_{i} n_{i}\right) \cdot \operatorname{dim}(\mathrm{Seg})-\sum_{i} n_{i}^{2}$, or approximately $(I+$ $\left.\sum_{i} n_{i}\right) \cdot \operatorname{dim}(\operatorname{Seg})$ for $\operatorname{dim}(\operatorname{Seg}) \gg n_{i}$. Because of the constraint on ISI (for example, indicating the absolute refractory period of muscle fibers), the number of discharges $n_{i}$ for each 
MU in a given active segment is limited. The complexity of each Tabu iteration is therefore linear w.r.t. the number of sources $I$ and the data length $\operatorname{dim}(\mathrm{Seg})$, in contrast to the NP-hardness of the space.

\section{Maximization on Two Segments}

The segment-by-segment maximization method, as described earlier, often converges to local rather than global maximal configurations of $\left(\boldsymbol{x}_{i}\right)_{i}$. It was experimentally observed that this phenomenon occurs when the following two conditions concur (see Section V-B for illustration): 1) two or more different MUs have similar action potential shapes; and 2) at least two consecutive segments are separated by a time interval smaller than $T_{R}$. Under these conditions, an error in the estimation of $\left(\boldsymbol{x}_{i}\right)_{i}$ in one segment is susceptible to yield a systematic estimation error on the adjacent segment. A solution to the early convergence problem is to maximize jointly on two consecutive segments using a similar algorithm structure of Tabu search as in Section III-C, if the two segments are separated by less than $T_{R}$.

For this purpose, we define the discharge patterns in two connected segments as a concatenated vector: $\boldsymbol{x}_{i}^{(k, k+1)}=$ $\left[\boldsymbol{x}_{i}^{(k)} ; \boldsymbol{x}_{i}^{(k+1)}\right]$, where $k$ and $k+1$ denote the segments index. $\boldsymbol{z}^{(k, k+1)}$ covers the interval of both segments and the in-between area of noises and $n_{i}^{(k, k+1)}=n_{i}^{(k)}+n_{i}^{(k+1)}$. Thus, the optimization function reads

$$
\begin{aligned}
& \underset{\left(\boldsymbol{x}_{i}\right)_{i}^{(k, k+1)}}{\arg \max } P\left(\left(\boldsymbol{x}_{i}\right)_{i}^{(k, k+1)} \mid\left(\boldsymbol{x}_{i}^{-(k, k+1)}, \boldsymbol{h}_{i}, m_{i}, \sigma_{i}^{2}\right)_{i}, \sigma_{\epsilon}^{2}, \boldsymbol{z}\right) \\
& =\underset{\left(\boldsymbol{x}_{i}\right)_{i}^{(k, k+1)}}{\arg \min } \frac{1}{2 \sigma_{\epsilon}^{2}}\left\|\boldsymbol{z}^{(k, k+1)}-\sum_{i=1}^{I} \boldsymbol{h}_{i} * 1_{\boldsymbol{x}_{i}}^{(k, k+1)}\right\|^{2} \\
& \quad+\sum_{i}^{I} \sum_{j=0}^{n_{i}^{(k, k+1)}} \frac{\left(\boldsymbol{x}_{i, j+1}^{(k, k+1)}-\boldsymbol{x}_{i, j}^{(k, k+1)}-m_{i}-T_{R}\right)^{2}}{2 \sigma_{i}^{2}} \\
& \quad+\log \sigma_{i} \sum_{i}^{I} n_{i}^{(k, k+1)} .
\end{aligned}
$$

Similarly to (16), $\boldsymbol{x}_{i, 0}^{(k, k+1)}$ and $\boldsymbol{x}_{i, n_{i}^{(k, k+1)}+1}^{(k, k+1)}$ denotes the last discharge in $\mathrm{Seg}_{k-1}$ and the first discharge in $\mathrm{Seg}_{k+2}$ respectively. The neighborhood definition can be easily generalized (see definition in Section III-C) on a concatenated version $\left(\boldsymbol{x}_{i}\right)_{i}^{(k, k+1)}$, allowing either to add, remove or relocate an existent MUAP across two segments. The neighborhood space is enlarged to $\left(I+\sum_{i} n_{i}^{(k, k+1)}\right)\left(\operatorname{dim}\left(\operatorname{Seg}_{k}\right)+\operatorname{dim}\left(\operatorname{Seg}_{k+1}\right)\right)$ from $\left(I+\sum_{i} n_{i}^{(k)}\right) \operatorname{dim}\left(\operatorname{Seg}_{k}\right)$. Note that for the two-segment approach the iterative maximization process is also carried out for each value of $k$.

With these modifications, a false label-switched configuration in one segment is no longer stable, since relocating an MUAP to the adjacent segment would further maximize (7). Comparisons are made in Section V-B on experimental EMG recordings to confirm that the two-segment maximization technique outperforms the one-segment version.

\section{VALIDATION METHODS}

Validation tests were conducted using a PC equipped with a 3.2-GHz Intel Pentium 4 CPU processor and a 3 G RAM.

\section{A. Simulation Model}

An EMG signal generation tool designed by Farina et al. [23] was used to simulate intramuscular EMG signals. In order to test the performance of the proposed decomposition algorithm, the following model parameter values were fixed (see 23 for details on the model).

1) Four to five MUs were activated in a single channel with $\Delta E_{\max }$ (the maximum normalized energy difference between MUAPs of different classes) set to 0.7.

2) Discharge rates of the active MUs were set in the range of $10-15 \mathrm{~Hz}$.

3) Maximum degree of overlapping between action potentials of different MUs was set as $\alpha_{\%, \max }=1$ (complete overlap).

4) Percentage of MUAPs in the interference signal which overlapped in time was set to $N_{\% s}=70 \%$.

5) $\mathrm{SNR}$ ratio was $\mathrm{SNR}_{\mathrm{dB}}=10$.

Five signals with the earlier properties were generated to test the proposed method. The sampling frequency of the simulated signals was $10 \mathrm{kHz}$. For both simulation and experimental tests in the following, the value of $T_{R}$ was fixed to $10 \mathrm{~ms}$, i.e. 100 samples for a $10 \mathrm{kHz}$ sampling frequency. Simulated signals had duration of $10 \mathrm{~s}$.

\section{B. Experimental Recordings}

Five healthy men (age, mean $\pm \mathrm{SD}=25.3 \pm 4.5$ years) participated in the study. The study was conducted in accordance with the Declaration of Helsinki, approved by the local Ethics Committee, and written informed consent was obtained from all subjects prior to participation.

The EMG measurements were obtained from the abductor digiti minimi muscle. The fifth finger was fixed in a custommade brace to record the force exerted during an isometric contraction of the muscle (Politecnico di Torino, Torino, Italy). The subjects performed three maximal voluntary contractions (MVCs) with the abductor digiti minimi, with each trial separated by a resting period of $2 \mathrm{~min}$. The greatest force was used as the reference MVC for the other contractions. A pair of wire electrodes made of Teflon coated stainless steel (A-M Systems, Carlsborg, WA; diameter $50 \mu \mathrm{m}$ ) was inserted into the muscle with a $25-\mathrm{G}$ needle. The insulated wires were cut to expose only the cross section at the tip. The needle was inserted to a depth of a few millimeters below the muscle fascia and removed to leave the wire electrodes inside the muscle. The intramuscular EMG signals were amplified and provided one bipolar recording (Counterpoint EMG, DANTEC Medical, Skovlunde, Denmark) that was bandpass filtered $(500 \mathrm{~Hz}-5 \mathrm{kHz})$ and sampled at $10 \mathrm{kHz}$. A reference electrode was placed around the wrist. Each subject performed two 60-s contractions at forces of $5 \%$ and $10 \% \mathrm{MVC}$ in random order and with $15 \mathrm{~min}$ of rest in between. 
As reference, the MUAPs were identified from the intramuscular recordings with a validated decomposition algorithm (EMGLAB) [8], [9]. This interactive algorithm includes a user interface for manually editing and verifying the results [9]. The EMGLAB displays a segment of the EMG signal, the templates of the action potentials of the identified MUs, the discharge patterns, and a close-up of the signal for resolving missed discharges and overlaps. Full, regular patterns provided confidence that the decomposition was correct, whereas gaps, extra discharges or uneven intervals provided an indication of possible decomposition errors. To assist in identifying missed discharges the program displays bars in the signal panel that indicate the expected discharge times of each MU. The signal portion can then be viewed in the close-up panel which displays the signal at an expanded scale and allows matching MU templates to be selected. The close-up panel also displays overlaps at an expanded scale which allows visual verification of the result and different sets of templates to be selected and adjusted to find the correct fit. Commands are also available for undoing identifications and deleting or merging templates. The acquired EMG signals were manually inspected with this user interface by an expert operator. The decomposition with EMGLAB has been previously cross-validated by decomposing on pairs of simultaneous EMG signals containing the same MU activities [24]. The discharge patterns estimated in this way were used as reference. To evaluate the performance of the proposed methods, we followed the accuracy criterion as introduced in [25] for the $i$ th MU train

$$
A(i)=\frac{N_{\text {Dis }}(i)-N_{\mathrm{FP}}(i)-N_{\mathrm{FN}}(i)}{N_{\mathrm{Dis}}(i)} \times 100 \%
$$

where $N_{\text {Dis }}(i)$ is the number of "true discharges" of the MU and $N_{\mathrm{FN}}(i)$ and $N_{\mathrm{FP}}(i)$ are, respectively, the number of false negatives and the number of false positives produced by the unsupervised decomposition algorithms for the $i$ th $\mathrm{MU}$ when compared to the reference from manual decomposition (the true discharges were assumed to be those extracted with manual decomposition with EMGLAB). An MU discharge identified by the proposed method was considered a correct estimate if it was detected within a window of $1 \mathrm{~ms}$ centered at the time of occurrence of the true discharge. It was also shown in [25] that by adopting

$$
A=\frac{1}{I} \sum_{i=1}^{I} A(i)
$$

as the overall decomposition accuracy, any MU train is of the same significance regardless of the MUAP shape, duration and number of discharges.

$\sigma_{i} / m_{i}<0.3$ corresponded to the limit of regularity a priori imposed (this limit can be changed by the user if necessary). Thus, among the MUs identified by the proposed method, those with $\sigma_{i} / m_{i}>0.3$ were automatically excluded from the analysis. The overall decomposition accuracy $A$ was thus averaged only on those $A(i)$ values corresponding to MUs under the condition that $\sigma_{i} / m_{i}<0.3$. Results for experimental signals were obtained from the decomposition of the first $10 \mathrm{~s}$ of the experimental recordings.

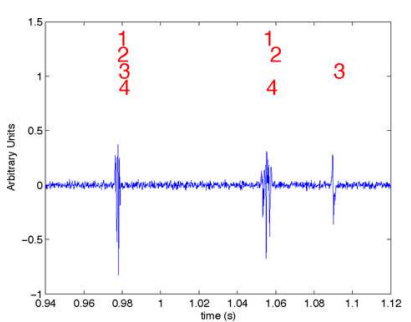

(a)

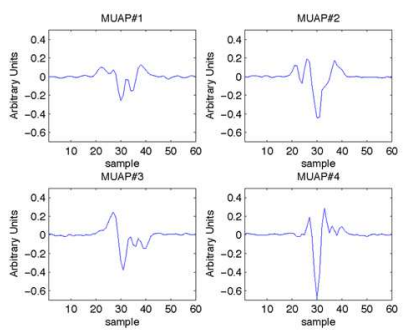

(c)

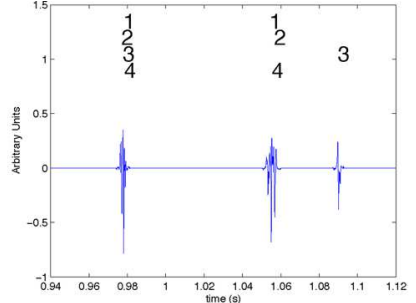

(b)

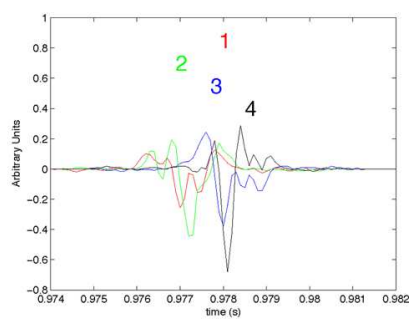

(d)
Fig. 1. Decomposition example using one-segment maximization on simulated EMG data. (a) Simulated EMG recordings and reference discharge pattern. (b) Reconstructed EMG and discharge pattern estimated by the decomposition method. No errors are present in this decomposition example. (c) Estimates of the MUAP shapes. (d) Four MUAPs overlapped in the first segment analyzed in this example on an expanded time scale.

TABLE I

DECOMPOSITION RESUlts ON SiMULATEd EMG SignAls (10 s)

\begin{tabular}{|c||c|c|c|c|c|}
\hline & EMG\#1 & EMG\#2 & EMG\#3 & EMG\#4 & EMG\#5 \\
\hline \# of MU & 5 & 5 & 4 & 4 & 4 \\
\hline \# of Val & 3 & 4 & 3 & 4 & 2 \\
\hline A & $88.7 \%$ & $88.0 \%$ & $92.1 \%$ & $88.5 \%$ & $94.6 \%$ \\
\hline
\end{tabular}

Note: The segment-by-segment Tabu search method is used. \# MU is the number of simulated MUs; \# Val is the number of MUs, which the proposed method extracted and validated according to the criterion on discharge variability.

\section{RESULTS}

\section{A. Simulations}

Fig. 1(a) shows a portion of a simulated signal. The MUAP shapes are illustrated in Fig. 1(c). The labels assigned to each MUAP are also displayed. In this example, the decomposition process took less than 5 min per iteration as described in steps 4(a) and (b) of the proposed method on an EMG signal of duration 10-s sampled at $10 \mathrm{kHz}$. Two iterations were run in this test and in all following results. Fig. 1(d) shows a segment with four overlapping MUAPs, in which the positive lobe of MUAP \#3 is compensated by the negative lobes of MUAP \#1 and MUAP \#2 (destructive overlap).

Table I shows the decomposition results on five simulated EMG signals. The MU discharge patterns from the decomposition were validated if $\sigma_{i} / m_{i}<0.3$, and the overall efficiency was calculated on validated MU sources. The one-segment maximization algorithm was sufficient for accurate decomposition of all simulated EMG signals.

\section{B. Experimental Signals}

In the experimental EMG signals recorded for 10\% MVC force, the total length (duration) of the active segments 


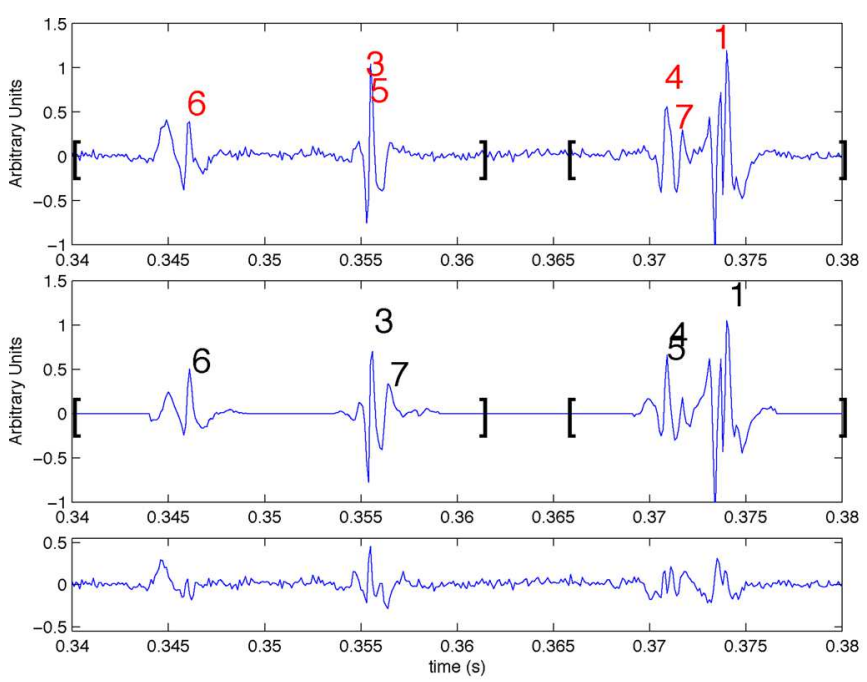

(a)

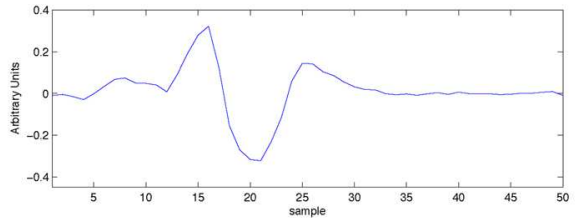

(c)

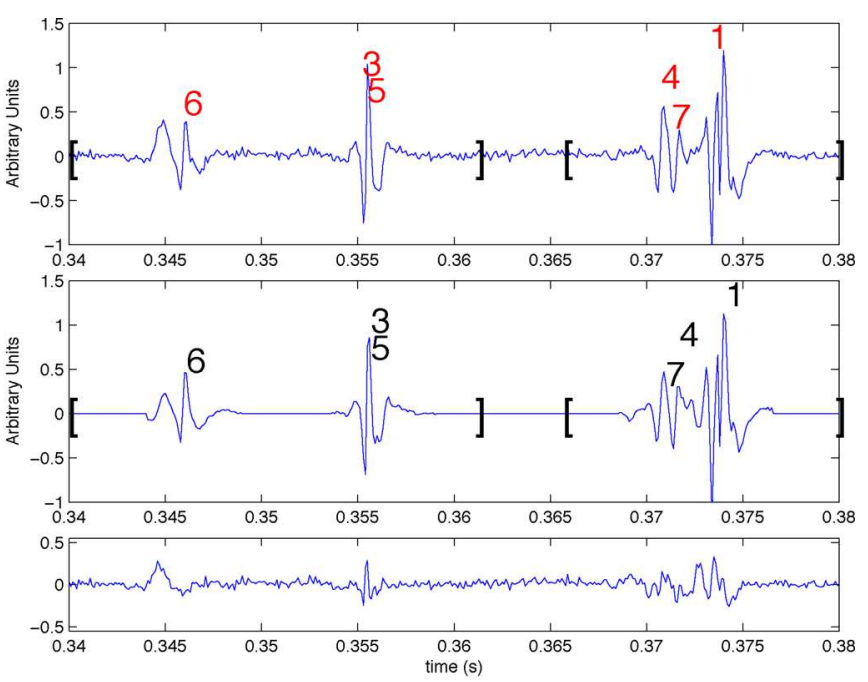

(b)

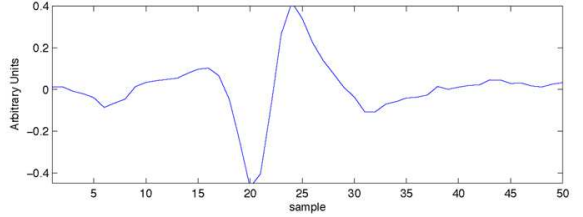

(d)

Fig. 2. Comparison of Tabu search within the one- and two-segment optimization approach. (a) Upper panel: EMG signal portion in which two segments are shown, delimited by square brackets. The reference MU labels are indicated with numbers as obtained by semiautomatic decomposition with an expert operator. Middle panel: The EMG signal reconstructed on the basis of the proposed automatic decomposition method applied to one-segment at a time. The estimated MU labels are shown and it can be observed that MU labels \#5 and \#7 are switched. Lower panel: The reconstruction error. (b) Same as in (a) but with the application of the two-segment optimization strategy. The MU labels are in this case all correctly estimated. Lower reconstruction error is observed while the MU discharge patterns remain regular. (c) and (d) MUAP shapes of the switched pair $\{\# 5, \# 7\}$.

corresponded to approximately $50 \%$ of the total recording duration when $8 \mathrm{MUs}$ were active. Among the active segments, those containing single MUAP (length inferior to $3 P$ ) accounted for approximately $50 \%$ of the total segments.

Contrary to the simulated signals, the one-segment maximization method was not optimal in the case of decomposition of the experimental EMG signals. Fig. 2 compares the two versions of the automatic decomposition method (one- and two-segment maximization) in the case of a $10 \%$ MVC EMG signal comprising seven active MUs. The MU labels in the upper panels of both Fig. 2(a) and (b) was obtained by trained experts using EMGLAB, while the middle panels show the reconstructed EMG signal based on the automatic decomposition method. In this example, the label switched pair $\{\# 5, \# 7\}$ in Fig. 2(a) appears across the two segments that are sufficiently close to each other (within $5 \mathrm{~ms}$ ); their MUAP shape estimates given in Fig. 2(c) and (d) show similar energy level. A local maximum of $\left(\boldsymbol{x}_{i}\right)_{i}^{(k)}$ includes MU\#7 instead of MU\#5 in Fig. 2(a) and this local configuration error is propagated to $\mathrm{Seg}_{k+1}$ since placing an MU\#7 in $\boldsymbol{x}_{7}^{(k+1)}$ would yield an unusually small ISI and penalize the posterior distribution through the prior laws on the discharge pattern of $\boldsymbol{x}_{7}$. The joint optimal solution on two segments is more "global" in a sense that the corresponding residual level is lowered, while the ISI regularity is almost unaltered, yielding a higher posterior probability [see Fig. 2(b)].
TABLE II

COMPARISON OF TWO VERSIONS OF OPTIMIZATION METHOD

\begin{tabular}{|c||c|c|c|c|c|}
\hline One-seg & MU\#1 & MU\#2 & MU\#3 & MU\#4 & MU\#5 \\
\hline FP & 26 & 117 & 63 & 112 & 115 \\
\hline FN & 22 & 65 & 34 & 42 & 66 \\
\hline TD & 146 & 118 & 122 & 89 & 135 \\
\hline A(i) & $67.2 \%$ & $\backslash$ & $21.5 \%$ & $\backslash$ & $\backslash$ \\
\hline$\sigma / m$ & 0.252 & 0.352 & 0.354 & 0.400 & 0.334 \\
\hline Val & 1 & 0 & 0 & 0 & 0 \\
\hline A & \multicolumn{5}{|c|}{$67.2 \%$} \\
\hline
\end{tabular}

\begin{tabular}{|c||c|c|c|c|c|}
\hline Two-seg & MU\#1 & MU\#2 & MU\#3 & MU\#4 & MU\#5 \\
\hline FP & 4 & 5 & 9 & 22 & 39 \\
\hline FN & 11 & 6 & 6 & 12 & 6 \\
\hline TD & 146 & 118 & 122 & 89 & 135 \\
\hline A(i) & $89.7 \%$ & $90.7 \%$ & $87.7 \%$ & $61.8 \%$ & $66.7 \%$ \\
\hline$\sigma / m$ & 0.256 & 0.224 & 0.215 & 0.303 & 0.311 \\
\hline Val & 1 & 1 & 1 & 0 & 0 \\
\hline A & \multicolumn{5}{|c|}{$89.4 \%$} \\
\hline
\end{tabular}

Note: The results of the one-segment optimization are reported in the upper table and those of the two-segment in the lower table. The decompositon was performed on a $10 \mathrm{~s}$ duration intramuscular EMG during a $10 \%$ MVC contraction. $\sigma / m<0.3$ is used as an automatic indicator of the validity of the decomposition results in all cases. " " indicates that the sum of false positives and false negatives exceeds the total number of true discharges.

To better illustrate the efficiency of the two-segment joint maximization method in comparison to the one-segment maximization method, decomposition statistics for each MU source are listed in Table II for one representative signal. Three MU discharge patterns are validated out of five for a two-segment joint maximization algorithm while only one of them is validated for the one-segment version. Some $A(i)$ are not cal- 


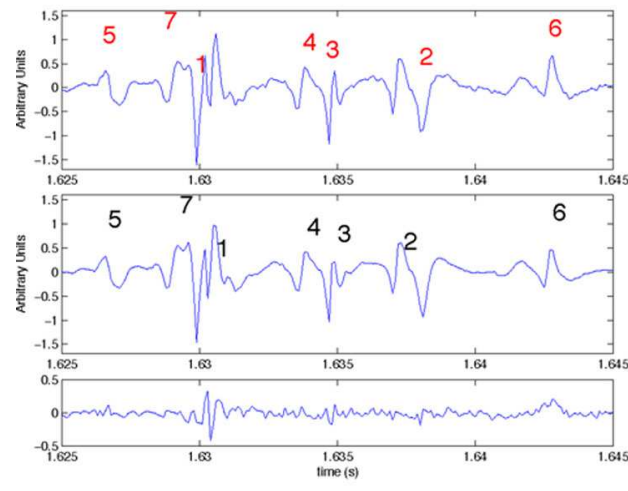

(a)

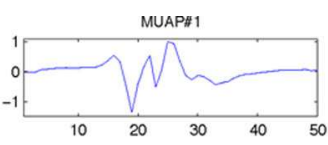

MUAP\#3
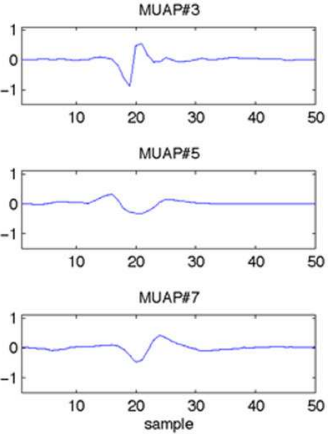
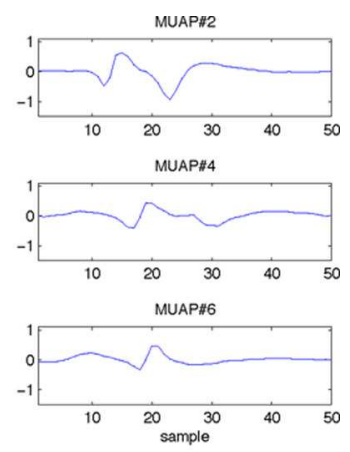

(b)

Fig. 3. (a) Upper panel: Segment of EMG and the MU labels decomposed by an expert operator. Middle panel: Signal reconstructed by the automatic decomposition method and the estimated MU labels. The proposed method estimated all labels correctly in this example. Lower panel: The reconstruction error. (b) Estimated MUAP shapes.

TABLE III

ACCURACY OF THE TWO-SEGMENT OPTIMIZATION ON EXPERIMENTAL SigNALS

\begin{tabular}{|c||c|c|c|c|c|}
\hline \multicolumn{1}{|c||}{} & \multicolumn{5}{c|}{ MVC = 5\% } \\
\cline { 2 - 6 } & EMG\#1 & EMG\#2 & EMG\#3 & EMG\#4 & EMG\#5 \\
\hline \# of MU & 4 & 7 & 5 & 5 & 4 \\
\hline \# of Val & 3 & 4 & 2 & 5 & 3 \\
\hline A & $96.4 \%$ & $87.7 \%$ & $96.2 \%$ & $88.7 \%$ & $88.6 \%$ \\
\hline \hline \multicolumn{5}{|c||}{} & \multicolumn{5}{c|}{ MVC = 10\% } \\
\cline { 2 - 6 } & EMG\#1 & EMG\#2 & EMG\#3 & EMG\#4 & EMG\#5 \\
\hline \# of MU & 5 & 5 & 8 & 8 & 4 \\
\hline \# of Val & 3 & 4 & 4 & 4 & 2 \\
\hline A & $89.4 \%$ & $85.1 \%$ & $87.7 \%$ & $87.2 \%$ & $92.5 \%$ \\
\hline Note: EMG signals are recorded during 5\% and $10 \%$ MVC contractions. The \# of Val is the
\end{tabular}
number of validated MU patterns for which $\sigma / m<0.3$.

culated as $N_{\mathrm{FP}}(i)+N_{\mathrm{FN}}(i)$ exceeds the grand total of true discharges for some sources in the first case. From these examples, it is evident that the decomposition quality of the twosegment maximization is superior to that of the one-segment maximization, while the computational time is less than doubled. The results also show that the two-segment optimization method corresponds to a better tradeoff between computation time and accuracy as a function of segment length for adjacent segments separated by less than $10 \mathrm{~ms}$ apart from each other as in Fig. 2. The accuracy would still improve by jointly optimizing the marginal posterior law w.r.t. all the segments (the entire EMG signal); however, the Tabu search computational load would be unacceptable in this case because the neighborhood complexity is dependent of the segment length (see discussions in Section III-C and III-D); at the other extreme, a segmentby-segment strategy requires less computational time but is less reliable (see Fig. 2). The following results on experimental signals were all obtained with the application of the two-segment optimization.

Fig. 3 reports an example of decomposition of a segment which contains seven MUs. This example shows that the method proposed is not limited by the number of overlapping MUAPs. Table III reports the results on all the analyzed experimental signals. Each column contains the total number of MU sources,

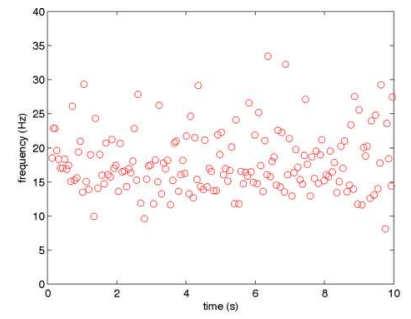

(a)

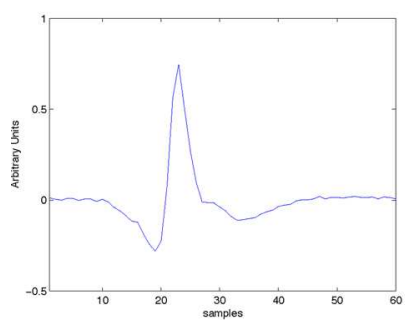

(c)

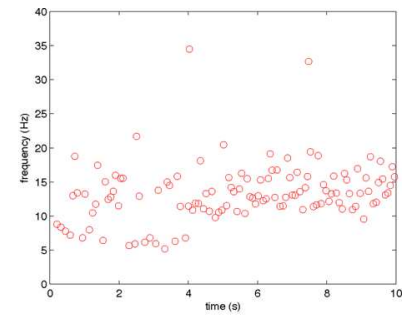

(b)

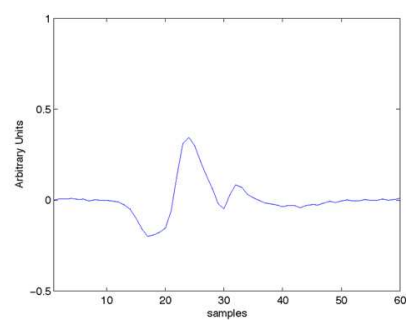

(d)
Fig. 4. (a) and (b) Instantaneous discharge rates obtained by the decomposition results on EMG of $10 \%$ MVC. (c) and (d) Corresponding MUAP shapes. (a) $\mathrm{FP}=0, \mathrm{FN}=3 /$ Total $=171$. (b) $\mathrm{FP}=1, \mathrm{FN}=12 /$ Total $=137$. (c) MUAP \#1. (d) MUAP \#2.

as identified by an expert operator, the number of validated MU discharge patterns and the overall accuracy rate $A$. No less than half of the MU sources were validated using the criterion $\sigma / m<0.3$ and $A$ was greater than $85 \%$ in all experimental results. The average accuracy was $91.5 \% \pm 4.4 \%$ and $88.4 \% \pm 2.8 \%$ for the signals recorded during $5 \%$ and $10 \%$ MVC contractions, respectively. The computational time required for the experimental signal decomposition (10-s duration) was in the range of 15-30 min, longer than for simulated signals mainly because of the higher discharge rates in experimental recordings.

The instantaneous discharge rates for the two validated MUs from the signal EMG \#5 at 10\% MVC (see Table III) are reported in Fig. 4(a) and (b), as estimated by the proposed automatic 
decomposition method. The estimated MUAP shapes are reported in Fig. 4(c) and (d). False-positive and false-negative statistics are also given in Fig. 4.

\section{DISCUSSION}

We have proposed a method for full decomposition of singlechannel intramuscular EMG signals that does not require any manual interaction. The attained accuracy approximates $90 \%$, compared to the results obtained by an expert operator with semiautomatic decomposition methods, for the case of contraction levels up to $10 \% \mathrm{MVC}$ and at most eight concurrently active MUs. In practical applications, this accuracy would be sufficient for the analysis of MU properties, such as the discharge rate and ISI variability. However, it may be insufficient to investigate physiological mechanisms for which a very precise decomposition is required, such as MU synchronization [26]. A manual decomposition may still be required in this case.

The proposed MAP estimator provides an efficient and fully unsupervised approach to cope with the decomposition of multiunit EMG signals. The Bayesian statistical model on the EMG data generation process allowed the inclusion of available prior information, such as the Gaussian-like distribution of ISIs, the refractory period of muscle fibers and the regularity in the spike discharge trains. The discharge pattern regularity has long been viewed as either a hint for the expert interaction or a posteriori evaluation criterion of a given decomposition method [8], [9], [17], and is now introduced into the completely automated decomposition method through the use of prior laws on $1_{\boldsymbol{x}_{i}}$. Although only general aspects of MU discharges are considered in this paper, further information could be integrated through the prior laws. The maximization of the posterior probability was achieved iteratively w.r.t. the continuous parameters and the NP-hard combinatorial discharge pattern. In addition, an extension of the segment-by-segment optimization method was introduced to treat the early convergence problem.

Some aspects of the method can be improved in future research. For example, the configurations of the Tabu algorithm remain an open problem, as to how to fix the FIFO memory length and the maximum number of iterations $M$, that in this paper has been empirically fixed to 20 . Another issue concerns the design of neighborhood of a given state. The computational load for each iteration grows as more configurations are included in the neighborhood, with better chances of avoiding local optima. The search space may also be increased by including optimal alignment of the action potentials [21] which is now limited by the sampling period. Finally, the proposed Bayesian decomposition approach is based on the modeling of a single-channel EMG recording. It is expected that a multichannel extension will improve the performance by exploiting the interchannel inference.

Concerning the statistical assumption on the independence of discharge patterns, we note that it does not constitute a limitation in the applicability of the approach but implies that the potential correlation between discharge patterns is not in- cluded in the prior laws. Thus, the method can still be applied to cases with high correlation between MU discharge patterns, such as tremor. In these cases, the correlation information is simply not exploited in the statistical model on which the method is based. In fact, the experimental signals were recorded from a muscle that presents relatively high degree of short-term synchronization between MUs [27]; thus, the assumption was not strictly valid in the analyzed experimental signals.

In conclusion, a new method for the decomposition of intramuscular EMG signals was proposed. It is fully automatic and provided an accuracy of approximately $90 \%$ on simulated signals and experimental single-channel recordings. The method does not make any assumptions on the particular shape of the action potentials or their spectral properties and can thus be applied to the spike classification problems of intraneural or intracortical recordings, for example.

\section{REFERENCES}

[1] C. J. De, Luca, R. S. LeFever, M. P. McCue, and A. P. Xenakis, "Control scheme governing concurrently active human motor units during voluntary contractions," J. Physiol., vol. 329, pp. 129-142, Aug. 1982.

[2] C. J. De, Luca, R. S. LeFever, M. P. McCue, and A. P. Xenakis, "Behaviour of human motor units in different muscles during linearly varying contractions," J. Physiol., vol. 329, no. 1, pp. 113-128, 1982.

[3] R. S. Lefever and C. J. De Luca, "A procedure for decomposing the myoelectric signal into its constituent action potentials: Part I. Technique, theory, and implementation," IEEE Trans. Biomed. Eng., vol. BME-29, no. 3, pp. 149-157, Mar. 1982.

[4] R. S. Lefever, A. P. Xenakis, and C. J. De Luca, "A procedure for decomposing the myoelectric signal into its constituent action potentials: Part II. Execution and test for accuracy," IEEE Trans. Biomed. Eng., vol. BME-29, no. 3, pp. 158-164, Mar. 1982.

[5] D. W. Stashuk, "Decomposition and quantitative analysis of clinical electromyographic signals," Med. Eng. Phys., vol. 21, no. 6/7, pp. 389-404, 1999.

[6] M. S. Lewicki, "Bayesian modeling and classification of neural signals," Neural Comput., vol. 6, pp. 1005-1030, 1994.

[7] F. A. Atiya, "Recognition of multiunit neural signals," IEEE Trans. Biomed. Eng., vol. 39, no. 7, pp. 723-729, Jul. 1992.

[8] K. C. McGill, "Optimal resolution of superimposed action potentials," IEEE Trans. Biomed. Eng., vol. 49, no. 7, pp. 640-650, Jul. 2002.

[9] K. C. McGill, Z. C. Lateva, and H. R. Marateb, "EMGLAB: An interactive EMG decomposition program," J. Neurosci. Methods, vol. 149, no. 2, pp. 121-133, Dec. 2005.

[10] A. Holobar and D. Zazula, "Correlation-based decomposition of surface EMG signals at low contraction forces," Med. Biol. Eng. Comput., vol. 42, no. 4, pp. 487-496, Jul. 2004.

[11] A. Holobar and D. Zazula, "Multichannel blind source separation using convolution kernel compensation," IEEE Trans. Signal Process., vol. 55, no. 9, pp. 4487-4496, Sep. 2007.

[12] M. Gazzoni, D. Farina, and R. Merletti, "A new method for the extraction and classification of single motor unit action potentials from surface EMG signals," J. Neurosci. Methods, vol. 136, no. 2, pp. 165-177, 2004.

[13] V. Dietz, E. Bischofberger, C. Wita, and H. J. Freund, "Correlation between the dischanges of two simultaneously recorded motor units and physiological tremor," Electroencephalogr. Clin. Neurophysiol., vol. 40, pp. 97-105, Jan. 1976.

[14] C. J. De Luca, "Physiology and mathematics of myoelectric signals," IEEE Trans. Biomed. Eng., vol. BME-26, no. 6, pp. 313-325, Jun. 1979 .

[15] N. Jiang, K. B. Englehart, and P. A. Parker, "A simulation method for the firing sequences of motor units," J. Electromyogr. Kinesiol., vol. 17, no. 5, pp. 527-534, 2007

[16] C. T. Moritz, B. K. Barry, M. A. Pascoe, and R. M. Enoka, "Discharge rate variability influences the variation in force fluctuations across the working 
range of a hand muscle," J. Neurophysiol., vol. 93, no. 5, pp. 2449-2459, May 2005.

[17] M. S. Lewicki, "A review of methods for spike sorting: The detection and classification of neural action potentials," Netw.: Comput. Neural Syst., vol. 9, no. 4, pp. R53-R78, 1998.

[18] R. Gut and G. S. Moschytz, "High-precision EMG signal decomposition using communication techniques," IEEE Trans. Acoust., Speech, Signal Process., vol. 48, no. 9, pp. 2487-2494, Sep. 2000.

[19] F. Glover, "Tabu search-Part I," ORSA J. Comput., vol. 1, pp. 190-206, 1989.

[20] K. C. McGill, K. L. Cummins, and L. J. Dorfman, "Automatic decomposition of the clinical electromyogram," IEEE Trans. Biomed. Eng., vol. BME-32, no. 7, pp. 470-477, Jul. 1985.

[21] K. C. McGill and L. J. Dorfman, "High-resolution alignment of sampled waveforms," IEEE Trans. Biomed. Eng., vol. BME-31, no. 6, pp. 462-468, Jun. 1984.

[22] S. Geman and D. Geman, "Stochastic relaxation, Gibbs distributions and the Bayesian restoration of images," IEEE Trans. Pattern Anal. Mach. Intell., vol. 6, no. 6, pp. 721-741, Nov. 1984.

[23] D. Farina, A. Crosetti, and R. Merletti, "A model for the generation of synthetic intramuscular EMG signals to test decomposition algorithms," IEEE Trans. Biomed. Eng., vol. 48, no. 1, pp. 66-77, Jan. 2001.

[24] K. McGill, Z. Lateva, and M. Johanson, "Validation of a computer-aided EMG decomposition method," in Proc. Eng. Med. Biol. Soc. (IEMBS 2004), 26th Аnnu. Int. Conf. IEEE, Sep., vol. 2, pp. 4744-4747.

[25] C. J. De Luca, A. Adam, R. Wotiz, L. D. Gilmore, and S. H. Nawab, "Decomposition of surface EMG signals," J. Neurophysiol., vol. 96, pp. 1646-1657, May 2006.

[26] M. A. Nordstrom, A. J. Fuglevand, and R. M. Enoka, "Estimating the strength of common input to human motoneurons from the crosscorrelogram," J. Physiol., vol. 453, pp. 547-574, 1992.

[27] D. Farina, C. Cescon, F. Negro, and R. M. Enoka, "Amplitude cancellation of motor-unit action potentials in the surface electromyogram can be estimated with spike-triggered averaging," J. Neurophysiol., vol. 100, no. 1, pp. 431-40, Jul. 2008.

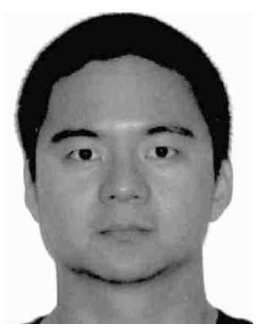

Di Ge received the B.S. degree in telecommunication from Jiaotong University, Shanghai, China, in 2003, the Diplôme d'Ingénieur from l'École Centrale de Nantes, Nantes, France, in 2006, where he is currently working toward the Ph.D. degree in signal processing at the Institut de Recherche en Communications et Cybernétique de Nantes, Unité Mixte de Recherche-Centre National de la Recherche Scientifique 6597, since 2006.

His current research interests include Bayesian ing inverse problems.

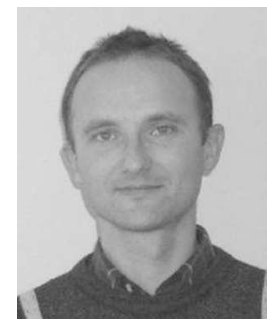

Eric Le Carpentier received the Ph.D. degree in automatic control from the Nantes University, Nantes, France, in 1990.

Since 1992, he has been an Assistant Professor at Ecole Centrale de Nantes, Nantes. His current research interests include signal processing, and currently focus on stochastic simulation for estimation, detection, tracking, and control, with applications in biomedical signals, robotics, and precise geolocation.

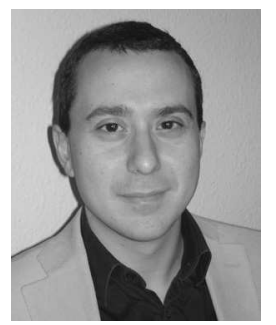

Dario Farina (M'01-SM'09) received the M.Sc. degree in electronics engineering from the Politecnico di Torino, Torino, Italy, in 1998, and the Ph.D. degree in automatic control and computer science and in electronics and communications engineering from the Ecole Centrale de Nantes, Nantes, France, and Politecnico di Torino, in 2002.

During 2002 to 2004, he was a Research Assistant Professor at Politecnico di Torino. During 20042008, he was an Associate Professor in biomedical engineering at Aalborg University, Aalborg, Denmark, where he has been a Full Professor in motor control and biomedical signal processing since 2008. He is a Reviewer of several scientific journals. He is a member of the Editorial Boards of the Journal of Neuroscience Methods, the Journal of Electromyography and Kinesiology, and the Medical and Biological Engineering and Computing, and a member of the Council International Society of Electrophysiology and Kinesiology (ISEK). He has authored or coauthored more than 160 papers in peer-reviewed journals. His current research interests include the areas of signal processing applied to biomedical signals, modeling of biological systems, basic and applied physiology of the neuromuscular system, neurorehabilitation technology, and brain-computer interfaces.

Dr. Farina is an Associate Editor of the IEEE TRANSACTIONS ON BIOMEDICAL ENGINEERING. 